\title{
Multimorbidität bei der geriatrischen Katze - nicht selten eine Herausforderung
}

\author{
Jürgen Kremendahl
}

Die Zahl der Katzenpatienten nimmt in vielen Kleintierpraxen ständig zu. Durch die vermehrte Aufmerksamkeit und den damit verbundenen medizinischen Fortschritt ist die Lebenserwartung der Katze in den letzten Jahrzehnten rasant gestiegen. Somit rücken geriatrische Erkrankungen dieser Spezies zunehmend in den Fokus der Kleintiersprechstunde. In dieser Übersichtsarbeit sollen die häufig vorkommenden Erkrankungen in ihren wechselseitigen Beziehungen vorgestellt werden, v.a. chronische Nierenerkrankung und Hyperthyreose sowie chronische Nierenerkrankung und Osteoarthritis.

\section{Einleitung}

Die Katze verdrängt in vielen Ländern den Hund als Nummer 1 auf der Beliebtheitsskala der Haustiere. In Deutschland ist ihr dies bereits vor vielen Jahren nachhaltig gelungen. Die Zahl der als Haustier gehaltenen Katzen hat sich in den letzten 15 Jahren nahezu verdoppelt, während die Zahl der gehaltenen Hunde nur vergleichsweise wenig variiert. Im Jahre 2014 standen 6,8 Millionen Hunde 11,8 Millionen Katzen gegenüber [9].

Dieser „Katzen-demografische“ Wandel hat vielfältige Gründe, die nicht zuletzt auf die veränderten Lebensumstände unserer eigenen Spezies zurückzuführen sind. Die Katze scheint auf den ersten Blick das ideale Haustier für unsere gegenwärtige Gesellschaft zu sein. Das kann sie in vielen Fällen sicher auch sein, wenn wir uns hin und wieder vergegenwärtigen, dass die Familie der Felidae zu den alleinlebenden Raubtieren gehört und mit Ausnahme der Löwen nicht in sozial strukturierten Gruppen lebt [6]. Flexibilität hat aber auch bei Katzen ihre Grenzen.
Auch in der Tiermedizin nimmt die Katze einen deutlich größeren Raum ein als in den vergangenen Jahrzehnten. Hierfür gibt es zahlreiche Gründe: Neben der zahlenmäßigen Zunahme der „Haustiger" und dem damit gestiegenen Interesse der Pharma- und Futtermittelforschung sowie -industrie, darf auch der Modellcharakter der Katze für die humane AIDS- und Lymphomforschung nicht außer Acht gelassen werden.

Die Lebenserwartung der Katze hat sich in diesen Jahrzehnten annähernd verdoppelt. Es sitzen täglich viele Katzensenioren im Wartezimmer. Auch hier ist eine Angleichung des Patienten „Katze“ an den Patienten „Mensch“ zu beobachten. Die Katze wird immer älter und die typischen Altersgebrechen nehmen zu. Einzeln diagnostiziert mögen diese Erkrankungen ein klares Therapieschema nach sich ziehen. Was ist aber, wenn mehrere Krankheiten gleichzeitig festgestellt werden, die sich im Verlauf beeinflussen und den Katzen, den Besitzern und nicht zuletzt den Tierärzten das Leben erschweren? Diese Übersicht konzentriert sich auf die fast täglich vorkommenden Krankheitskombinationen. Sie erhebt in keinster Weise einen Anspruch auf Vollständigkeit.

\section{Hyperthyreose und chronische Nierenerkrankung}

Diese Kombination ist sicher die häufigste Kombination geriatrischer Erkrankungen in der Katzensprechstunde ( $\bullet$ Tab. 1). Die chronische Nierenerkrankung der Katze (CNE) kann nicht nur die Diagnose der Hyperthyreose erschweren, sie beeinflusst auch deren Therapie und Prognose.

Über 10\% der hyperthyreoten Katzen leiden zum Zeitpunkt der Diagnose ihrer
Schilddrüsenerkrankung bereits unter einer chronischen Nierenerkrankung. Ob es einen kausalen Zusammenhang zwischen den beiden Erkrankungen gibt, kann bisher nicht mit Sicherheit entschieden werden. In jedem Fall ist die Interaktion der beiden Krankheiten komplex.

Es werden verschiedene Möglichkeiten diskutiert, durch welche Mechanismen eine Hyperthyreose das Entstehen einer chronischen Nierenerkrankung verursachen oder dazu beitragen kann. Diskutiert wird ein tubulointerstitieller Schaden, der in der Folge zu Fibrose und chronischer interstitieller Nephritis führt und durch einen Anstieg von Angiotensin II verursacht wird. Weiterhin werden Mikroinfarkte mit resultierender Fibrose als Folge einer Hypertension, die bei einem Teil der hyperthyreoten Katzen diagnostiziert werden kann, diskutiert [7].

Eine Hyperthyreose kann durch eine Erhöhung der glomerulären Filtrationsrate (GFR) für einen gewissen Zeitraum eine bereits existierende chronische Nierenerkrankung „maskieren“. Die Nierenwerte scheinen besser als sie in Wirklichkeit sind.

\section{Diagnose}

Katzen, die an beiden Erkrankungen leiden, können zur diagnostischen Herausforderung werden.

\section{Euthyroid-Sick-Syndrom}

Eine feline chronische Nierenerkrankung geht nicht selten mit einem EuthyroidSick-Syndrome (ESS) einher, also einer Veränderung der Schilddrüsenhormone durch eine Nicht-Schilddrüsenerkrankung. 
Tab. 1 Ergebnisse der Blutuntersuchung einer multimorbiden älteren Katze mit Hyperthyreose, chronischer Nierenerkrankung, chronischer Pankreatitis und derzeit in Remission befindlichem Diabetes mellitus. Der Diabetes mellitus wurde iatrogen durch Kortikoide zur Behandlung eines eosinophilen Granuloms ausgelöst. Die Katze konnte seit 6 Jahren erfolgreich gemanaged werden.

\begin{tabular}{|c|c|c|c|}
\hline Parameter & Einheit & Ergebnis & Referenzwert \\
\hline \multicolumn{4}{|l|}{ Blutbild } \\
\hline Leukozyten & $\mathrm{G} / \mathrm{l}$ & 6,5 & $6-11$ \\
\hline Erythrozyten & $\mathrm{T} / \mathrm{I}$ & 10,2 & $5-10$ \\
\hline Hämoglobin & $\mathrm{g} / \mathrm{dl}$ & 14,5 & $9-15$ \\
\hline Hämatokrit & $\%$ & 47 & $28-45$ \\
\hline MCV & fl & 46 & $40-55$ \\
\hline $\mathrm{MCH}$ & $\mathrm{pg}$ & 14 & $13-17$ \\
\hline $\mathrm{MCHC}$ & $\mathrm{g} / \mathrm{dl}$ & 31 & $31-35$ \\
\hline Thrombozyten & $\mathrm{G} / \mathrm{l}$ & 233 & $150-550$ \\
\hline Retikulozyten (relativ) & $\%$ & 0,25 & - \\
\hline Retikulozyten (absolut) & $|\mu|$ & 26000 & $<50000$ \\
\hline \multicolumn{4}{|l|}{ Differenzialblutbild } \\
\hline basophile Granulozyten & $\%$ & 0 & $0-1$ \\
\hline eosinophile Granulozyten & $\%$ & 2 & $0-6$ \\
\hline Segmentkernige & $\%$ & 79 & $50-75$ \\
\hline Lymphozyten & $\%$ & 16 & $15-50$ \\
\hline Monozyten & $\%$ & 3 & $0-4$ \\
\hline basophile Granulozyten (absolut) & $|\mu|$ & 0 & $\leq 200$ \\
\hline eosinophile Granulozyten (absolut) & $|\mu|$ & 104 & $0-600$ \\
\hline Segmentkernige (absolut) & $|\mu|$ & 5137 & $3000-11000$ \\
\hline Lymphozyten (absolut) & $|\mu|$ & 1022 & $1000-6000$ \\
\hline Monozyten (absolut) & $|\mu|$ & 201 & $0-500$ \\
\hline LUC $^{*}$ & $\%$ & 0 & - \\
\hline \multicolumn{4}{|l|}{ Niere } \\
\hline Harnstoff-N & $\mathrm{mg} / \mathrm{dl}$ & 39 & $16-38$ \\
\hline Kreatinin & $\mathrm{mg} / \mathrm{dl}$ & 1,9 & $<1,9$ \\
\hline Natrium & $\mathrm{mmol} / \mathrm{l}$ & 151 & $147-159$ \\
\hline Chlorid & $\mathrm{mmol} / \mathrm{l}$ & 115 & $109-129$ \\
\hline Kalium & $\mathrm{mmol} / \mathrm{l}$ & 2,8 & $3,3-5,8$ \\
\hline anorganisches Phosphat & $\mathrm{mmol} / \mathrm{l}$ & 1,1 & $0,8-2,2$ \\
\hline \multicolumn{4}{|l|}{ Leber } \\
\hline Bilirubin & $\mathrm{mg} / \mathrm{dl}$ & 0,2 & $<0,4$ \\
\hline $\mathrm{ALT}(\mathrm{GPT})$ & $\mathrm{U} / \mathrm{I}$ & 68 & $<175$ \\
\hline alkalische Phosphatase & $\mathrm{U} / \mathrm{l}$ & 39 & $<73$ \\
\hline$Y-G T$ & $\mathrm{U} / \mathrm{l}$ & $<1$ & $<5$ \\
\hline AST (GOT) & $\mathrm{U} / \mathrm{I}$ & 33 & $<71$ \\
\hline GLDH & $\mathrm{U} / \mathrm{I}$ & 2 & $<11$ \\
\hline Gesamteiweiß & $\mathrm{g} / \mathrm{dl}$ & 7,9 & $5,9-8,7$ \\
\hline Albumin im Serum & $\mathrm{g} / \mathrm{dl}$ & 3,2 & $2,7-4,4$ \\
\hline Globulin & $\mathrm{g} / \mathrm{dl}$ & 4,7 & $2,9-5,4$ \\
\hline Albumin-Globulin-Quotient & - & 0,69 & $>0,57$ \\
\hline \multicolumn{4}{|l|}{ Pankreas } \\
\hline Glukose & $\mathrm{mg} / \mathrm{dl}$ & 283 & $63-140$ \\
\hline Cholesterin & $\mathrm{mg} / \mathrm{dl}$ & 254 & $<329$ \\
\hline Fruktosamin & $\mu \mathrm{mol} / \mathrm{l}$ & 228 & $190-365$ \\
\hline \multicolumn{4}{|l|}{ Muskel } \\
\hline CK & $\mathrm{U} / \mathrm{I}$ & 144 & $<542$ \\
\hline $\mathrm{LDH}$ & $\mathrm{U} / \mathrm{I}$ & 144 & $<182$ \\
\hline Kalzium & $\mathrm{mmol} / \mathrm{l}$ & 2,5 & $2,2-2,9$ \\
\hline Magnesium & $\mathrm{mmol} / \mathrm{l}$ & 0,9 & $0,6-1,1$ \\
\hline Triglyceride gesamt & $\mathrm{mg} / \mathrm{dl}$ & 73 & $21-432$ \\
\hline \multicolumn{4}{|l|}{ Schilddrüsenprofil } \\
\hline T4 (Gesamtthyroxin) & $\mu \mathrm{g} / \mathrm{dl}$ & 4,4 & $0,87-4,7$ \\
\hline freies Thyroxin & $\mathrm{ng} / \mathrm{dl}$ & 3,7 & $0,7-2,6$ \\
\hline \multicolumn{4}{|l|}{ feline spezifische Pankreaslipase } \\
\hline Ergänzungstest & $\mu g / l$ & 18 & $\leq 3,5$ \\
\hline
\end{tabular}


Spricht die Symptomatik deutlich für eine Hyperthyreose, sollte die Verdachtsdiagnose auch bei einem normalen Gesamt-T4 (TT4) hinterfragt werden.

Es gibt verschiedene Möglichkeiten, um den Verdacht zu sichern oder auszuräumen: Da der Thyroxinspiegel variiert, reicht es in einem Teil der Fälle, den Test nach 3-4 Wochen zu wiederholen. Zudem besteht die Möglichkeit, im Labor den freien-T4-Wert (fT4) bestimmen zu lassen. Die Ergebnisse der TT4- und fT4Tests sollten gemeinsam interpretiert werden: Ein TT4 in der oberen Hälfte des Referenzbereichs in Zusammenhang mit einem erhöhten fT4 macht das Vorliegen einer Schilddrüsenüberfunktion sehr wahrscheinlich. Ein erhöhter fT4 mit einem niedrigen TT4 in der unteren Hälfte des Referenzbereichs macht eine Hyperthyreose hingegen recht unwahrscheinlich. Bei der Durchführung des Tests ist eine Gleichgewichtsdialyse aufgrund der größeren Genauigkeit vorzuziehen, wenngleich die Untersuchung mit einem erhöhten Kostenaufwand verbunden ist.

Die weiteren diagnostischen Möglichkeiten werden selten benötigt:

- Bestimmung des Thyroidea stimulierenden Hormons (TSH)

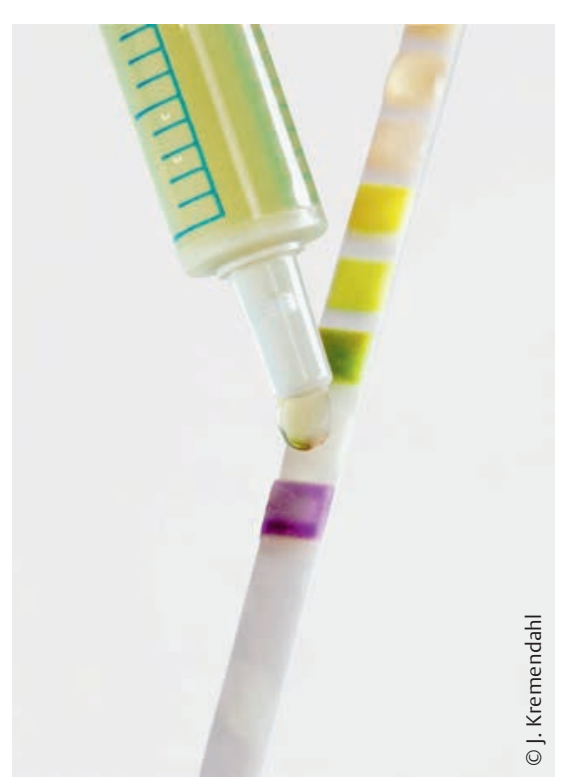

Abb. 1 Urinuntersuchungen inkl. bakteriologischer Kultur von steril gewonnenem Punktionsurin gehören zur Basisdiagnostik der meisten geriatrischen Katzenkrankheiten.
- Triiodthyronin-Suppressionstest (T3Suppressionstest)

- Thyreotropin-Releasing-Hormone-Stimulationstest (TRH-Stimulationstest)

- Szintigrafie der Schilddrüse

Während der T3-Suppressions- und der TRH-Stimulationstest in ihrer Durchführung aufwendig sind, steht die Szintigrafie der Schilddrüse nur in spezialisierten Einrichtungen zur Verfügung.

\section{Hyperthyreose}

Vergleichsweise einfach hingegen ist die Interpretation eines Blutbefunds mit einem Kreatinin und einem TT4, die beide im oberen Referenzbereich angesiedelt sind. Es ist zu vermuten, dass die Katze an einer Hyperthyreose und einer chronischen Nierenerkrankung leidet.

Durch die Hyperthyreose sind die Kreatininwerte häufig „falsch“ erniedrigt bzw. maskiert. Kreatinin ist ein Endprodukt des Muskelstoffwechsels. Dies erklärt, warum die typischerweise dünne, wenig muskulöse hyperthyreote Katze einen eher niedrigen Kreatininspiegel hat.

In der Regel wird in der Nierendiagnostik neben dem Kreatinin- auch auf den Harnstoffwert zurückgegriffen. Dieser kann sowohl bei chronischer Nierenerkrankung als auch bei der Hyperthyreose erhöht sein, da durch den gesteigerten Appetit vermehrt Protein aus der Nahrung aufgenommen wird. Eine Harnstofferhöhung bei einer (nüchternen) älteren Katze reicht alleine also nicht, um den Verdacht auf eine chronische Nierenerkrankung zu untermauern. Bei beiden Werten kann eine erhöhte glomeruläre Filtrationsrate im Rahmen einer Hyperthyreose zu reduzierten Blutspiegeln führen.

Es bleibt abzuwarten, inwieweit sich der Symmetric-Dimethylarginine-Test (SDMA-Test) als hilfreich bei der chronischen Nierenerkrankung und der Kombination chronische Nierenerkrankung und Hyperthyreose erweisen wird. Der Test, der in absehbarer Zeit in Deutschland eingeführt werden soll, soll eine nachlassende Nierenfunktion deutlich früher im Routinelabor sichtbar machen als dies bisher möglich war [5].
Auch bei der unabdingbaren Urinuntersuchung gibt es 3 wesentliche Auffälligkeiten, die bei beiden Krankheiten zu finden sind:

- Erniedrigung des spezifischen Gewichts

- unterschiedlich ausgeprägte Proteinurie

- häufigere Inzidenz von Harnwegsinfekten $(\triangleright$ Abb. 1)

\section{Therapie}

Für die meisten Katzen, bei denen eine Therapie gegen die Hyperthyreose eingeleitet wird, hat die Behandlung keine Nierenprobleme zur Folge. Die Tiere können die deutliche Reduktion des renalen Blutflusses und damit der glomerulären Filtrationsrate kompensieren. Bei einem Teil der Katzen kommt es unter der Therapie jedoch zur Dekompensation. Der reduzierte renale Blutfluss „demaskiert“ in der Folge eine bis dahin latente chronische Nierenerkrankung. Die Prozentangaben hierzu schwanken in der Literatur erheblich und reichen bis $\mathrm{zu} 1 / 3$ der betroffenen Katzen. Bisher ist es mithilfe der oben geschilderten diagnostischen Tests nicht möglich, die Katzen, die dieses Problem entwickeln, vorab zu identifizieren. Die Bestimmung der glomerulären Filtrationsrate könnte hierfür hilfreich sein. Sie wird aber vermutlich ebenso wie einige andere Nierenmarker - wegen des aufwendigen Testprotokolls keine Praxisrelevanz erlangen.

Es ist davon auszugehen, dass sich die glomeruläre Filtrationsrate ca. 4 Wochen nach Einstellung der Schilddrüse eingependelt hat.

Eine Hyperthyreose sollte zunächst medikamentös therapiert werden, bevor dauerhafte (und nicht reversible) Therapieoptionen wie eine Thyreoidektomie oder eine Radioiodtherapie erwogen werden.

Es scheint empfehlenswert, zur Sicherheit einige Monate länger zu warten, insbesondere wenn das orale Thyreostatikum (Thiamazol, Carbimazol) problemlos vertragen wird.

Bei Katzen, die unter oraler Therapie nicht tolerable Nebenwirkungen ent- 
wickeln, kann eine transdermale Applikation von Thiamazolsalbe versucht werden. Voraussetzung hierfür ist die Umwidmung in einem Therapienotstand gemäß § 56a, Abs. 2 (4) des Arzneimittelgesetzes (AMG). Der Vorteil der transdermalen Applikation liegt im sanften, verzögerten Wirkungseintritt. Die Salbe eignet sich besonders bei hyperthyreoten Katzen, bei denen man schon zu Therapiebeginn eine chronische Nierenerkrankung vermutet. Erfreulicherweise stehen inzwischen auch unterschiedlich hoch dosierte orale Thiamazolpräparate zur Verfügung. Der Tierarzt muss somit keine unnötig hohen Anfangsdosen verordnen, die in der Lage wären, eine Dekompensation der Nieren zu forcieren.

Wenn beide Erkrankungen gleichzeitig vorliegen, ist das optimale Therapieergebnis für den individuellen Patienten oft nur durch häufige Dosisanpassung des Thyreostatikums zu erreichen. Die pragmatische Überlegung des Therapeuten lautet: Was braucht mein Patient derzeit mehr? Hat die Katze einen akzeptablen TT4, aber recht hohe Nierenwerte, die mit einer Inappetenz einhergehen, reduziert der Autor die Dosis des Thyreostatikums und beobachtet, ob sich die Nierenwerte verbessern und der Appetit zunimmt. Oder macht die Schilddrüse mehr Probleme? Nimmt die Katze sehr gut Futter auf und verliert trotzdem Gewicht, sodass die Niere Nahrungs- und Muskeleiweiß verstoffwechseln muss, korrigiert der Autor die Dosis des Thyreostatikums nach oben.

Natürlich müssen für ein optimales Therapieergebnis auch die CNE-Richtlinien der International Renal Interest Society (IRIS) in die Behandlung integriert werden [2].

Häufig ist bei den Patienten eine engmaschige Überwachung der relevanten Blutwerte nötig, bis die Tiere einen relativ stabilen Zustand erreichen. Trotz der prinzipiell ungünstigen Kombination der Erkrankungen kann bei umsichtiger Therapie in den meisten Fällen ein gutes Gleichgewicht erzielt werden, sodass den Katzen über lange Zeit eine gute Lebensqualität erhalten bleiben kann.

\section{Osteoarthritis und chronische Nierenerkrankung}

Die Osteoarthritis ist eine chronischprogressive Erkrankung, bei der der Gelenkknorpel langsam zerstört wird und der betroffene Knochenabschnitt mit Umbauprozessen und Osteophytenzubildung reagiert. Die Erkrankung, die bei der Katze meist ab einem Alter von 10 Jahren auftritt, ist am häufigsten an Schulter-, Ellbogen- und Hüftgelenk lokalisiert.

Hauptsymptome sind:

- reduzierte Aktivität

- Bewegungsunlust

- Lahmheiten

- Inappetenz

- nachlassende Fellpflege

- Unsauberkeit

- Aggressivität

- Verstopfung

- Alopezie über den betroffenen Gelenken

\section{Diagnose}

Es ist wichtig, den klinischen Verdacht einer Osteoarthritis röntgenologisch abzusichern, da sich die Symptome zum Teil durchaus mit denen einer kognitiven Dysfunktion oder Senilität überschneiden, deren Inzidenz ebenfalls mit zunehmendem Alter steigt. Beispielhaft seien hier Aggressivität und Unsauberkeit angeführt.

\section{.konkret}

Bei Osteoarthritis sollte eine Therapieentscheidung immer auf Klinik und Bildgebung beruhen.

Eine ausgeprägte, röntgenologisch diagnostizierte Spondylose kann klinisch irrelevant sein und sollte - insbesondere bei chronischer Nierenerkrankung nicht automatisch zu einer antientzündlichen Therapie führen.

\section{Therapie}

Die folgenden Therapieansätze sind zur Behandlung einer Osteoarthritis und einer gleichzeitig vorliegenden chronischen Nierenerkrankung geeignet:

- Gewichtsreduktion

- Chondroprotektiva 


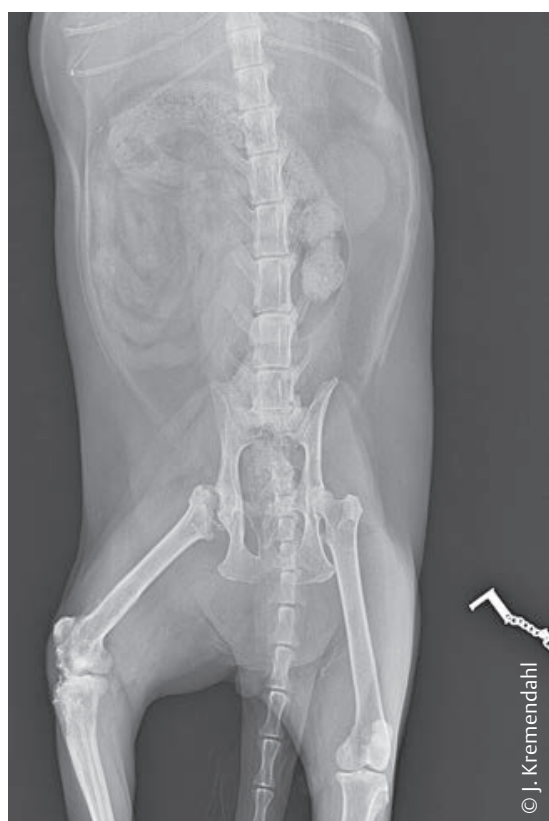

Abb. 2 Röntgenbild eines 17-jährigen, männlich-kastrierten EKH-Katers. Aufgrund hochgradiger Veränderungen am rechten Knie und an der rechten Hüfte braucht dieser Patient trotz seiner chronischen Nierenerkrankung eine konsequente Schmerztherapie.
- Nahrungsergänzungsmittel

- ggf. alternative Therapieansätze wie Akupunktur, Magnetfeldtherapie, biologische und homöopathische Einzeloder Komplexmittel

\section{NSAIDs}

Anders verhält es sich beim Einsatz von nicht steroidalen Antiphlogistika (NSAIDs). Hier gilt es - nicht zuletzt auch wegen der zunehmenden Sensibilisierung der Katzenhalter - sorgfältig abzuwägen, ob die Anwendung eines NSAID sinnvoll ist. Aufgrund einer Warnung der Food and Drug Administration (FDA) in den USA aus dem Jahre 2010 wird regelmäßig auf die Verschreibung von Meloxicam Bezug genommen [8]. Diese Warnung wird häufig in einschlägigen Foren diskutiert. Konkret ging es dabei darum, dass Meloxicam nicht wiederholt als Injektionspräparat bei der Katze und dass die orale Hundesuspension nicht bei Katzen angewendet werden darf. Dies ist darauf zurückzuführen, dass Fälle von aku- tem Nierenversagen bei Katzen in den USA vorgekommen seien. Wiederholte Injektionen sind noch immer nicht zugelassen. Seit eine orale Suspension in verminderter Konzentration für Katzen mit passender Dosierhilfe erhältlich ist, ist das Risiko von Dosierungsfehlern deutlich gesunken.

NSAIDs haben insbesondere über die Hemmung der Cyclooxygenase 1 (COX-1) Einfluss auf die Prostaglandinsynthese. Prostaglandine haben einen gastroprotektiven Effekt und spielen eine wichtige Rolle bei der Aufrechterhaltung des renalen Blutflusses, insbesondere bei Hypotension und Dehydratation. Die Reduktion des Prostaglandinspiegels erklärt somit häufige Nebenwirkungen bei COX1-Hemmern.

Meloxicam ist COX-1-sparend, die Halbwertzeit ähnelt der beim Hund. Es bietet sich daher als Langzeitpräparat an. Das Präparat ist zudem in flüssiger Form mit 
hoher Akzeptanz verfügbar, was eine genaue Dosierung und die Bestimmung der niedrigsten effektiven Dosis erleichtert. Eine Studie von Gowan et al. (2011) zeigte, dass Meloxicam in einer oralen, täglichen Dosis von $0,02 \mathrm{mg} / \mathrm{kg}$ älteren Katzen mit Osteoarthritis dauerhaft gegeben werden kann, auch wenn diese an chronischer Nierenerkrankung leiden [4]. Bei einigen Katzen der Studie, die an chronischer Nierenerkrankung litten, schien die Langzeitgabe von Meloxicam sogar die Progression der chronischen Nierenerkrankung zu verlangsamen. Natürlich sollte eine unter chronischer Nierenerkrankung leidende Katze nur nach abgesicherter Osteoarthritis-Diagnose, bei entsprechendem Leidensdruck und nach gescheiterten Therapieversuchen mit den oben genannten anderen Optionen eine Meloxicam-Therapie beginnen ( Abb.2). Nach Ansicht des Verfassers muss im Zweifelsfall die Lebensqualität der Katze an erster Stelle stehen.

Ein Behandlungsversuch mit einem NSAID wie Meloxicam ist bei entsprechender Klinik - anfänglich unter engmaschiger Laborkontrolle - gerechtfertigt.

Ein Nierenprofil (Kreatinin, Harnstoff, Phosphat) sollte dabei nach etwa 4 Wochen durchgeführt werden. Je nach Ausgangswert und Entwicklung der Werte unter der Therapie können die Kontrollen auf ein 3-monatiges Intervall ausgedehnt werden.

Robenacoxib ist ein selektiver COX2-Hemmer, der ein ähnlich günstiges Profil wie Meloxicam aufweist. Der Wirkstoff ist bisher nur zur kurzfristigen Anwendung bis zu 6 Tagen zugelassen. Über die Halbwertzeit der NSAIDs Carprofen, Ketoprofen und Tolfenaminsäure ist wenig bekannt. Sie sind in Deutschland nur für eine Kurzzeitbehandlung der Katze zugelassen.

\section{Kortikoide und Opioide}

Sollte sich eine Verschlechterung der Nierenwerte zeigen, die auf das NSAID zurückzuführen ist, stehen Kortikoide wie Prednisolon und Opioide wie Buprenorphin als weitere Behandlungsoptionen zu Verfügung. Prednisolon ist zwar entzündungshemmend, aber auch potenziell knorpelschädigend und heilungsverzögernd, sodass es nur nach Ausschöpfung der anderen Therapieoptionen zur Anwendung kommen sollte.

\section{Antikonvulsivum}

Gabapentin, eigentlich ein Antikonvulsivum, könnte in Zukunft eine größere Rolle bei der Schmerztherapie, v.a. auch bei Neuropathien der Katze spielen. Bisher gibt es allerdings nur eine bislang unveröffentlichte Studie zur Wirkung von Gabapentin bei der Osteoarthritis der Katze, die allerdings positive Effekte feststellen konnte [3].

\section{Weitere Ko-Morbiditäten}

Es gibt eine Reihe weiterer Erkrankungen bei der geriatrischen Katze, die sich in der Diagnostik oder der Therapie beeinflussen, aber in der täglichen Praxis nicht so häufig vorkommen. Als Beispiele hierfür seien exemplarisch noch 2 weitere Kombinationen aufgeführt: Bei einem Diabetes mellitus und einer Hyperthyreose überschneiden sich die Symptome wie Polyphagie, Gewichtsverlust, Polydipsie und Polyurie weitgehend. Beide Erkrankungen treten aber selten simultan auf und sind - zumindest auf Laborebene - leicht zu differenzieren.

Bei chronischer Nierenerkrankung und gleichzeitiger chronischer Pankreatitis kann es schwierig werden, eine optimale Diätempfehlung zu geben. Bezieht eine Nierendiät ihre Energie durch die Proteinreduktion im hohen Maße aus Fett, ist eine Reduktion des Fettanteils bei der chronischen Pankreatitis vorteilhaft.

Vorsicht ist ebenfalls geboten, wenn eine Operation oder Narkose bei einer geriatrischen Katze erforderlich wird. Dies ist zum Beispiel bei einer relevanten, schmerzhaften Zahnerkrankung der Fall.

Veränderter Stoffwechsel, nachlassendes Immunsystem sowie kardiale Vorerkrankungen müssen zu einem individuellen Narkoseprotokoll führen.

Das Alter stellt keine Kontraindikation für einen operativen Eingriff dar. Nach 
entsprechender Aufklärung zeigen sich die meisten Patientenbesitzer einsichtig und stimmen dem Eingriff zu.

\section{Fazit}

Die Geriatrie wird bei Katze und Hund in Zukunft einen immer größeren Platz in der täglichen Kleintiersprechstunde einnehmen. Die in dieser Übersicht genannten Beispiele belegen, dass die geriatrische Katzenmedizin eine durchaus anspruchsvolle Disziplin sein kann. In vielen Fällen kann die Lebenszeit bei zufriedenstellender Lebensqualität - also sinnvoll - verlängert werden. Dies ist ein guter Grund, sich auch auf diesem Gebiet über neue Erkenntnisse zu informieren.

\section{Online zu finden unter}

http://dx.doi.org/10.1055/s-0041-107652

\section{Literatur}

1 August JR. Consultations in Feline Internal Medicine. $6^{\text {th }}$ ed. St. Louis: Saunders Elsevier; 2010

2 Elanco Animal Health. Im Internet: http:// www.iris-kidney.com/; Stand: Oktober 2015

3 Epstein M, Rodan I, Griffenhagen G et al. Im Internet: http://www.aaha.org/public_ documents/professional/guidelines/2015_ aaha_aafp_pain_management_guidelines_ for_dogs_and_cats.pdf; Stand: Oktober 2015
4 Gowan RA, Lingard AE, Johnston L et al. Retrospective case-control study of the effects of long-term dosing with meloxicam on renal function in aged cats with degenerative joint disease. J Feline Med Surg 2011; 13 (10): 752-761

5 IDEXX Laboratories, Inc. Im Internet: https:/| www.idexx.com/small-animal-health/ solutions/articles/sdma-diagnose-kidneydisease.html; Stand: Oktober 2015

6 International cat care. Im Internet: http:/| icatcare.org/advice/understanding-your-cat/ social-structure-cat-life; Stand: Oktober 2015

7 Little S. The Cat: Clinical Medicine and Management. St. Louis: Saunders Elsevier; 2011

8 U.S. Food and Drug Administration. Im Internet: http://www.fda.gov/AnimalVeterinary/ NewsEvents/CVMUpdates/ucm231254.html; Stand: Oktober 2015

9 Statista GmbH Hamburg. Im Internet: http:// de.statista.com/statistik/daten/studie/30157/ umfrage/anzahl-der-haustiere-in-deutschenhaushalten-seit-2008/; Stand: Oktober 2015

Dr. Jürgen Kremendahl

Berghauser Str. 128

42349 Wuppertal

praxis@dr-kremendahl.de 